\title{
Basic Concept on Asymptotes in Calculus
}

Jagat Krishna Pokhrel*

\begin{abstract}
In analytical geometry an asymptote of a curve is a line such that the distance between the curve and the line approaches zero as one or both of the $x$ or $y$ coordinates tend to infinity. Some source includes the requirements that the curve may not cross the line infinitely often but this is unusual for modern definition. In some content such as algebraic geometry an asymptote is defined as a line which is tangent to a curve at infinity.

In some case a curve may have a branch or branches extending beyond the finite region. In this case of $p$ be a point on such a branch of the curve, having its coordinates $(x, y)$ and if $P$ moves along the curve, so that at least one of $x$ and $y$ tend to $+\infty$ and to $-\infty$, then $P$ is said to tend to infinites and this we denote by $P \rightarrow \infty$.
\end{abstract}

Key words: Vertical, Horizontal, Oblique- asymptote, infinite, Tangent, Coordinates

\section{Definition}

A straight line is said to be an asymptote of a curve $\mathrm{y}=\mathrm{f}(\mathrm{x})$, if the perpendicular distance of the paint $\mathrm{p}(\mathrm{x}, \mathrm{y})$ on the curve from the line tends to 0 when $\mathrm{x}$ or $\mathrm{y}$ or both tend to infinity.

We shall consider separately the case which rise when an asymptotes (i) parallel to either coordinate axis (ii) Parallel to neither. An asymptote parallel to y-axis may be referred as a "vertical asymptote and parallel to x-axis as a horizontal asymptote. An asymptote which is not parallel to either axis may be described as an oblique asymptote that is, for curves given by the graph of a function $\mathrm{y}=\mathrm{mx}+\mathrm{c}$, horizontal asymptote are horizontal lines that the graph of the function approaches as $\mathrm{x}$ tends to $+\infty$ or $-\infty$. Vertical asymptote is vertical line near which the function grows without bound. An oblique asymptote has a slope that is non-zero but finite, such that graph of the function approaches its as $\mathrm{x}$ tends to $+\infty$ or $-\infty$.

\section{Objectives}

To get the better feeling for the techniques and terminology used in it and so far, discussed the graph of some function and relevant reading is focused on identifying vertical, horizontal and oblique asymptote and predicted the behavior

* Mr. Pokhrel is associate Professor in Mathamatics in T.U. 


\section{Review of literature}

The word asymptotes is derived from Greek which means not falling together, from “together" 'follen' (Oxford Dictionary (1989), the term was introduced by Apollonius of perga in his work on conic section but in contrast to its modern meaning used it to mean any line that does not intersect the curve ( Smith, Dover) (1958), p. 318

Asymptotes convey information about the behavior of curve in the large and determining the asymptotes of a function is an important step in sketching its graph (Apostal-1967) the study of asymptotes of functions, constructed in a broad sense, forms a part of the subject of Asymptotic Analysis.

\section{Methodology}

To find the asymptotes, commonly encountered in the study of calculus are of curves of the form $\mathrm{y}=\mathrm{mx}+\mathrm{c}$ these can be computed using limits and classified in to horizontal, vertical and oblique asymptotes depending on their orientation. Horizontal asymptotes are horizontal line that the graph of the function approaches as $\mathrm{x}$ tends to $+\infty$ or $-\infty$. As the name indicates they are parallel to the $\mathrm{x}$-axis. Vertical asymptotes are vertical line (perpendicular to $\mathrm{x}$-axis) near which the function grows without bound. Oblique asymptotes are diagonal lines such that the difference between the curve and the line approaches 0 as $x$ tends to $+\infty$ to $-\infty$. More general types of asymptotes can be defined in this case. Only open curves that have some infinite branch can have an asymptote. No closed curve can have an asymptote (Coddington, Earl 1989)

\section{Asymptotes Parallel to y-axis (Vertical Asymptotes)}

Let $\mathrm{x}=\mathrm{k}$ be an asymptote of the curve $\mathrm{y}=\mathrm{f}(\mathrm{x})$ parallel to $\mathrm{y}$-axis. The distance PM of a paint $\mathrm{p}(\mathrm{x}, \mathrm{y})$ on the curve from the line $\mathrm{x}=\mathrm{k}$ is $|\mathrm{x}-\mathrm{k}|$. Also, $\mathrm{y}$ tends to $+\infty$ or $-\infty$ as the curve tends to be asymptotic to the line $\mathrm{x}=\mathrm{k}$. (Santi Narayan Represented 1988). Which is shown in fig 1

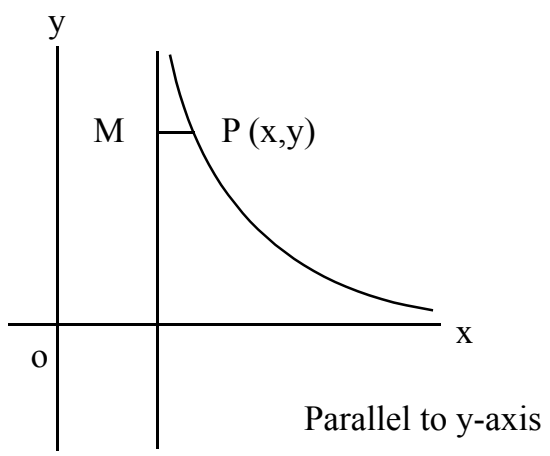

Fig-1 
Thus, in order that $\mathrm{x}=\mathrm{k}$ be an asymptote of the curve $\mathrm{y}=\mathrm{f}(\mathrm{x})$, we have the following possibilities.
(i) $\lim _{x \rightarrow \infty(k+0)} y=+\infty$
(ii) $\lim _{x \rightarrow \infty(k+0)} y=+\infty$
(iii) $\lim _{x \rightarrow \infty(k+0)} y=-\infty$

(iv) $\lim _{x \rightarrow \infty(k+0)} y=-\infty$

We can display as in the fig.

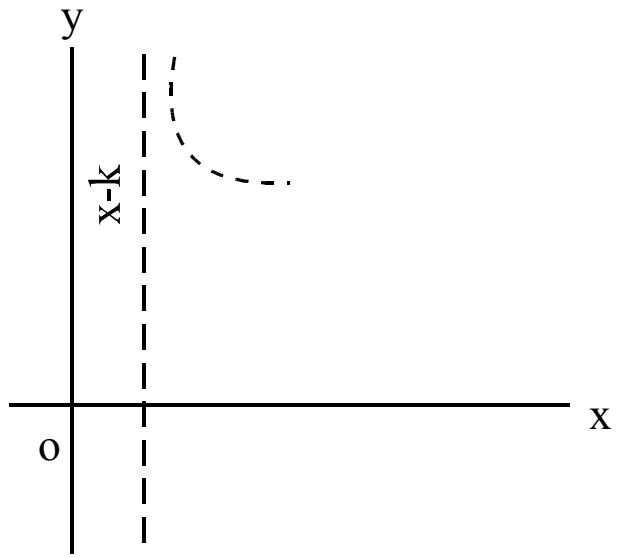

(i)

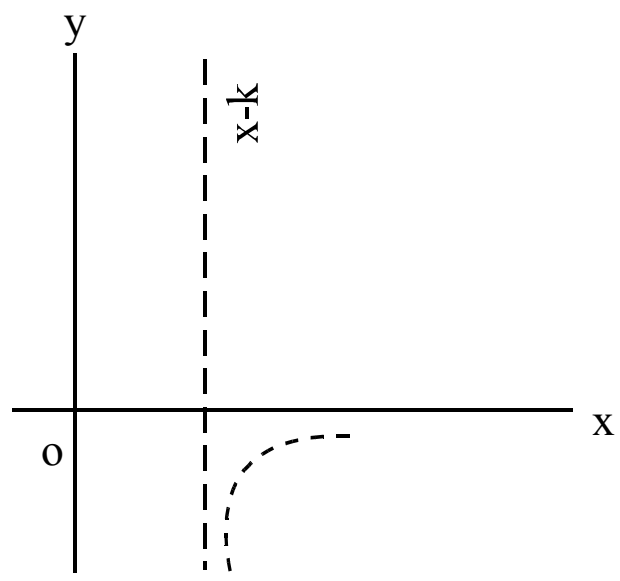

(iii)

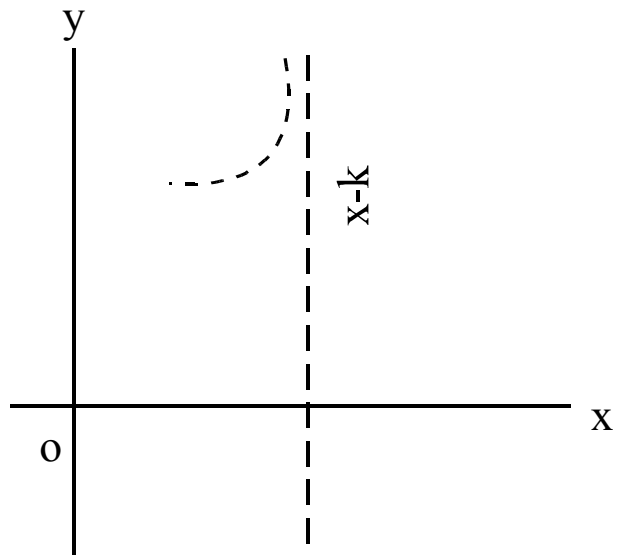

(ii)

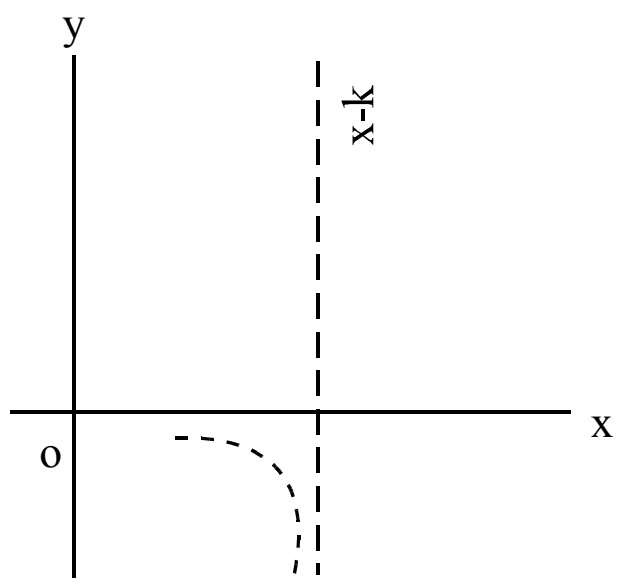

(iv) 


\section{Asymptotes parallel to a-axis (Horizontal Asymptotes)}

Let $\mathrm{y}=\mathrm{k}$ be an asymptote parallel to $\mathrm{x}$-axis of the curve $\mathrm{y}-\mathrm{f}(\mathrm{x})$

The distance PM of a paint $\mathrm{P}(\mathrm{x}, \mathrm{y})$ on the curve from the line $\mathrm{y}=\mathrm{k}$ is $|\mathrm{y}-\mathrm{k}|$ Also, $\mathrm{x} \rightarrow$ $+\infty$ or $-\infty$ as the curve tends to be asymptotic to the line $y=k$. Shown in fig- 2

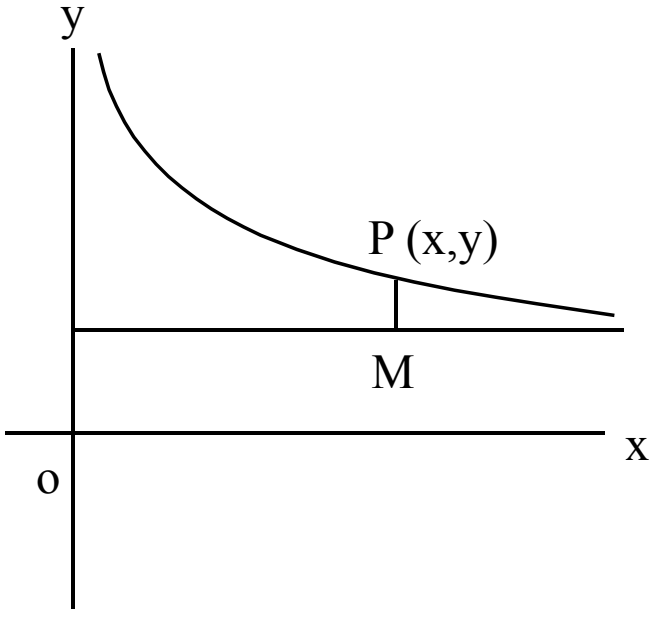

Fig-2, parallel to $\mathrm{x}$-axis

Again, we have the possibilities

i) $\lim _{x \rightarrow+\infty} y=k$ (ii) $\lim _{x \rightarrow-\infty}=k$

as $\mathrm{x} \rightarrow+\infty$, y may tend to $\mathrm{k}$ from values greater than or less than $\mathrm{k}$. Similarly, as $\mathrm{n} \rightarrow$ $-\infty$ may tend to $\mathrm{K}$ from values greater than or less than $\mathrm{K}$.

So, there are four possibilities which are displayed as in the fig.
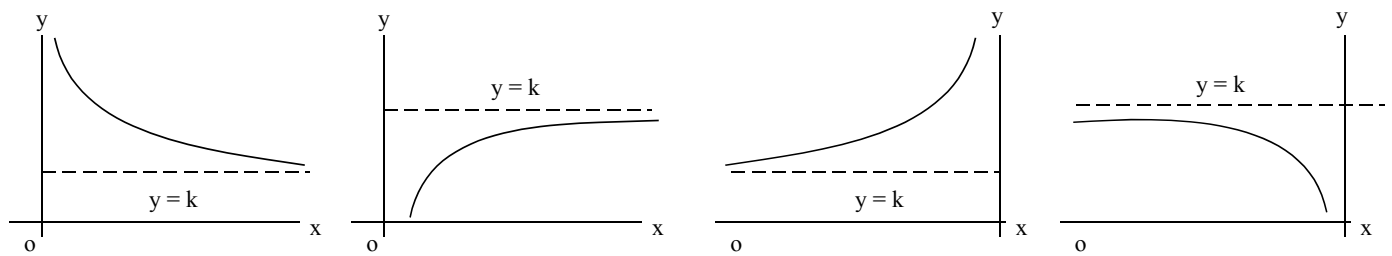

Thus, when $\mathrm{x} \rightarrow+\infty$ and $\mathrm{y}$ tends $\mathrm{k}$ from the values greater than $\mathrm{K}$, we say that the curve while going to the right tend to the asymptote from above.

Oblique asymptotes: let $y=m x+c$ be an asymptote of $y=f(x)$. The perpendicular distance $\mathrm{PM}$ of a paint $\mathrm{P}(\mathrm{x}, \mathrm{y})$ on the curve from the line $\mathrm{y}=\mathrm{mx}+\mathrm{c}$ is 


$$
\frac{|y-m x-c|}{\sqrt{1+m^{2}}}
$$

Which $\rightarrow 0$ as $\mathrm{x}$ tends to $+\infty$ or $-\infty$. Thus $\mathrm{y}-\mathrm{mx}-\mathrm{c} \Rightarrow 0$ as $\mathrm{x} \rightarrow \pm \infty$

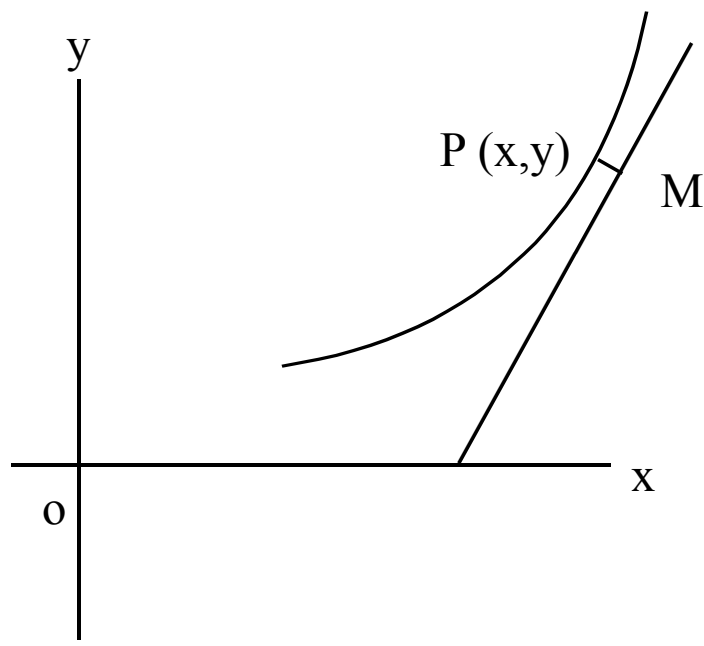

Fig 3

Asymptotes of algebraic curves: The most useful case of determination of asymptotes is for algebraic curves. The general form of the equation of an algebraic curve of the nth degree is arranging in groups of homogeneous terms (Das and Mukherjee) (1986).

$\left(a_{0} x^{n}+a_{1} x^{n-1} y+a_{2} x^{n-2} y^{2}+\ldots \ldots+a_{n} y^{n}\right)+\left(b_{0} x^{n-1}+b_{1} x^{n-2} y+\ldots \ldots+b_{n-1} y^{n-1}\right)+\left(\operatorname{cox}{ }^{n-2}+\right.$ $\left.\mathrm{c}_{1} \mathrm{x}^{\mathrm{n}-3} \mathrm{y}+\ldots \ldots+\mathrm{c}_{\mathrm{n}-2} \mathrm{y}^{\mathrm{n}-2}\right)+\ldots \ldots=0 \ldots \ldots$ (a)

Which can also be written as

$\mathrm{x}^{\mathrm{n}} \phi_{\mathrm{n}}(\mathrm{y} / \mathrm{x})+\mathrm{x}^{\mathrm{n}-1} \phi_{\mathrm{n}-1}(\mathrm{y} / \mathrm{x})+\mathrm{x}^{\mathrm{n}-2} \phi_{\mathrm{n}-2}(\mathrm{y} / \mathrm{x})+\ldots=0$

Where $\phi r$ is an algebraic polynomial of degree $r$. For asymptotes of this curve, we can prove given below,

\section{Rule (a) for the -Asymptotes not parallel to y-axis}

let the curve of Asymptote not parallel to $\mathrm{y}$ axis will be $\mathrm{y}=\mathrm{mx}+\mathrm{c}$, where $\mathrm{m}$ is any of the real finite roots of $\phi_{n}(m)=0$ and for each such value of $m, c=-\phi(m) / \phi_{n-1}(m)$ provided it gives a definite value of $\mathrm{c}$. 
Proof:

let the equation $\mathrm{x}^{\mathrm{n}} \phi_{\mathrm{n}}(\mathrm{y} / \mathrm{x})+\mathrm{x}^{\mathrm{n}-1} \phi_{\mathrm{n}-1}(\mathrm{y} / \mathrm{x})+\mathrm{x}^{\mathrm{n}-2} \phi_{\mathrm{n}-2}(\mathrm{y} / \mathrm{x})+\ldots . .=0$ o the curve can be put in the form $\phi_{\mathrm{n}}(\mathrm{y} / \mathrm{x})+\ln \phi_{\mathrm{n}-1}(\mathrm{y} / \mathrm{x})+\frac{1}{x^{2}} \phi_{\mathrm{n}-2}(\mathrm{y} / \mathrm{x})+\ldots \ldots \ldots . .=0$----- (1) Now, if $\mathrm{y}=\mathrm{mx}+\mathrm{c}$ be an asymptote, where $\mathrm{m}$ and $\mathrm{c}$ are finite $\mathrm{ln}_{\mathrm{n} \rightarrow \infty}^{\mathrm{t}}(\mathrm{y} / \mathrm{x})=\mathrm{m}$. Hence from (1) making $\mathrm{x} \rightarrow \infty$ since $\mathrm{m}$ is finite, and the function $\phi \mathrm{n}(\mathrm{m}), \phi_{\mathrm{n}-1}(\mathrm{~m})$ etc. which are algebraic polynomials in $\mathrm{m}$ are accordingly finite, we get $\phi_{\mathrm{n}}(\mathrm{m})=0$ (Goyal J.K., Gupta, K.P. 1999)

Again, Since in this case ${ }_{\mathrm{n} \rightarrow \infty}^{\mathrm{lt}}(\mathrm{y}-\mathrm{mx})=\mathrm{c}$, e can write $\mathrm{y}-\mathrm{mx}=\mathrm{c}+\mathrm{u}$ where $\mathrm{u}$ is a function of $\mathrm{x}$ such that $\mathrm{u} \rightarrow 0$ when $\mathrm{x} \rightarrow \infty$. Thus $\frac{y}{x}=\mathrm{m}+\mathrm{c}+\frac{c+u}{x}$

Now from (i), we get

$\phi \mathrm{n}\left(\mathrm{m}+\frac{c+u}{x}\right)+\frac{1}{x} \phi_{\mathrm{n}-1}\left(\mathrm{~m}+\frac{c+u}{x}\right)+\frac{1}{x^{2}} \phi_{\mathrm{n}-2}\left(\mathrm{~m}+\frac{c+u}{x}\right)+\ldots \ldots=0$

Expanding each term by Taylor's theorem, since the function $\phi_{\mathrm{r}}$ are all algebraic polynomials and will each lead to a finite series and remembering that $\phi_{\mathrm{n}}(\mathrm{m})=0$, we get

$$
\begin{aligned}
& \left\{\frac{c+u}{x} \phi_{n}(m)+\frac{(c+u)^{2}}{x^{2} \cdot 2 !} \phi^{\prime \prime}(m)+\frac{(c+u)^{3}}{x^{3} \cdot 3 !} \phi^{\prime \prime} n(m)+\ldots . .\right\}^{\prime}+ \\
& \frac{1}{x}\left\{\phi_{n-1}(m)+\frac{c+u}{x} \phi_{n-1}^{\prime}(m)+\frac{(c+u)^{2}}{x^{2} \cdot 2 !} \phi^{\prime \prime}{ }_{n-1}(m)+\ldots . .\right\}+ \\
& \frac{1}{x^{2}}\left\{\phi_{n-2}(m)+\frac{c+u}{x} \phi_{n-2}^{\prime}(m)+\ldots . .\right\}+\ldots . .=0
\end{aligned}
$$

Now multiplying throughout by $\mathrm{x}$ and making $\mathrm{x} \rightarrow \infty$, we get

$(\therefore \mathrm{u} \rightarrow 0), \mathrm{c} \phi^{\prime}{ }_{\mathrm{n}}(\mathrm{m})+\phi_{\mathrm{n}-1}(\mathrm{~m})=0$ or $\mathrm{c}=-\phi_{\mathrm{n}-1}(\mathrm{~m}) / \phi^{\prime} \mathrm{n}(\mathrm{m})$.

Each finite root of $\phi_{\mathrm{n}}(\mathrm{m})=0$ will give one value of c provided $\phi^{\prime}(\mathrm{m}) \neq 0$ for this value and accordingly we get the corresponding asymptote $\mathrm{y}=\mathrm{mx}+\mathrm{c}$. 
Rule (b) for the-Asymptotes parallel to y-axis: Asymptotes parallel to y-axis exists only when an, the coefficient of the highest power of $y$ i.e. of $y^{n}$ is zero and in this case the coefficient of the highest available power of $y$ in the equation provided it involve $\mathrm{x}$ and is not merely a constant equated to zero will give us those asymptotes (Gupta and Mittal- 2000).

Proof: A similar rule will apply to asymptotes parallel to $\mathrm{x}$-axis.

After dividing by $\mathrm{y}^{\mathrm{n}}$ and replacing $1 / \mathrm{y}$ by $\mathrm{z}$ the equation.

$\left.\left(a_{0} x^{n}+a_{1} x^{n-1} y+a_{2} x^{n-2} y^{2}+\ldots . .+a_{n} y^{n}\right)+b o x^{n-1}+b_{1} x^{n-2} y+\ldots+b_{n-1} y n^{-1}\right)+\left(c o x^{n-2}+c_{1}\right.$ $\left.\mathrm{x}^{\mathrm{n}-3} \mathrm{y}+\ldots . .+\mathrm{c}_{\mathrm{n}-2} \mathrm{y}^{\mathrm{n}-2}\right)+\ldots \ldots=0$

of the curve can be written in ascending powers of $\mathrm{z}$ in the form

$$
a_{n}+z\left(a_{n-1} x+b_{n-1}\right)+z^{2}\left(a_{n-2} x^{2}+b_{n-2} x+c_{n-2}\right)+\ldots .=0----
$$

This will have an asymptote parallel to $\mathrm{y}-$ axis of the type $\mathrm{x}=\mathrm{a}$ where is finite, provided $\mathrm{z} \rightarrow 0$ when $\mathrm{x} \rightarrow \mathrm{a}+0$.

Hence making $\mathrm{z} \rightarrow 0$, since $\mathrm{x}$ now tends to a finite value. We must have the necessary condition $\mathrm{a}_{\mathrm{n}}=0$

Assuming this to be satisfied, we get from (1) dividing by $\mathrm{z}$ and making $\mathrm{z} \rightarrow 0$

$$
a_{n-1} x+b_{n-1}=0
$$

Giving a finite value of $x$ provided $a_{n-1}$ is not zero which makes $|y| \rightarrow \infty$ and thus represents an asymptote. In case $a_{n-1}$ is also zero along with an in order that we may have an asymptote parallel to $y$-axis, since $x$ is to be finite, we must have from (3) $\mathrm{b}_{\mathrm{n}-1}=0$ Hence from (1) dividing by $\mathrm{z}^{2}$ and making $\mathrm{z} \rightarrow 0$ now, we get the asymptotes parallel to $\mathrm{y}$-axis given by

$a_{n-2} x^{2}+b_{n-2} x+c_{n-2}=0$. Provided this gives finite value of $x$. In case $a_{n-1}, b_{n-2}, c_{n-2}$ are identically zero, we proceed in a similar manner with the coefficient of $z^{2}$ in (3) i.e. the coefficient of $y^{n-3}$ in the original equation (1) and so on providing the rule.

\section{The working rule for asymptote of algebraic curves}

For an algebraic curve of the nth degree with equation given by (a) as given above, first of all if the term involving $\mathrm{y}^{\mathrm{n}}$ is absent in which case, the coefficient of the highest power of $y$ involved in the unless it is merely a constant independent of $x$ equated to zero will give asymptotes parallel to y-axis. Similarly, if the term involving 
$\mathrm{x}^{\mathrm{n}}$ is absent, the coefficient of the highest available power of $\mathrm{x}$ equation to zero will in general give asymptotes parallel to the x-axis. (Das \& Mukherjee 1986).

Next, replacing $x$ by 1 and $y$ by $m$ in the homogenous $n^{\text {th }}$ degree terms, get $\phi_{n}(m)$ [as is apparent from the alternative form $\mathrm{x}^{\mathrm{n}} \phi_{\mathrm{n}}(\mathrm{y} / \mathrm{x})$. Similarly get $\phi_{\mathrm{n}-1}(\mathrm{~m})$ from the $(\mathrm{n}-1)$ th degree terms and if necessary $\phi_{\mathrm{n}-2}(\mathrm{~m})$ from the $(\mathrm{n}-2)^{\text {th }}$ degree terms and so on. Now equating $\phi_{\mathrm{n}}(\mathrm{m})$ to zero, obtain the real finite roots $\mathrm{m}_{1}, \mathrm{~m}_{2}$ etc which will indicate the direction of the corresponding asymptotes repeated roots giving in general a set of parallel asymptotes.

For each non repeated roots $m_{1}$ say, a definite value $c_{1}$ of $c=-\phi_{n-1}(m) / \phi_{n}^{\prime}(m)$ is obtained and the corresponding asymptotes $y=m_{1} x+c_{1}$ is determined. For repeated roots, the several values of $\mathrm{c}$ may be obtained.

\section{Asymptote of polar curves}

Let $r=f(\theta)$ be the polar equation of the curve, this may be written as $u=1 r=1 \mathrm{f} \theta$ $=\mathrm{F}(\theta$

$\mathrm{P}$ being any paint $(\mathrm{r}, \theta)$ on the curve, $\mathrm{P} \rightarrow \infty$ as $\mathrm{r} \rightarrow \infty$ which requires $\mathrm{F}(\theta) \rightarrow 0$. Let the solution of $F(\theta)=0$ be $\theta=\alpha, \beta, \gamma \ldots$ etc. (Singh and Bajracharya (2055) then these are the only directions along which the branches of infinity)

Consider the branch corresponding to $\theta=\alpha$. Let the straight line $\gamma \cos \left(\theta-\alpha_{1}\right)=\mathrm{P} \ldots \ldots \ldots$. (2) Be the asymptote to this branch

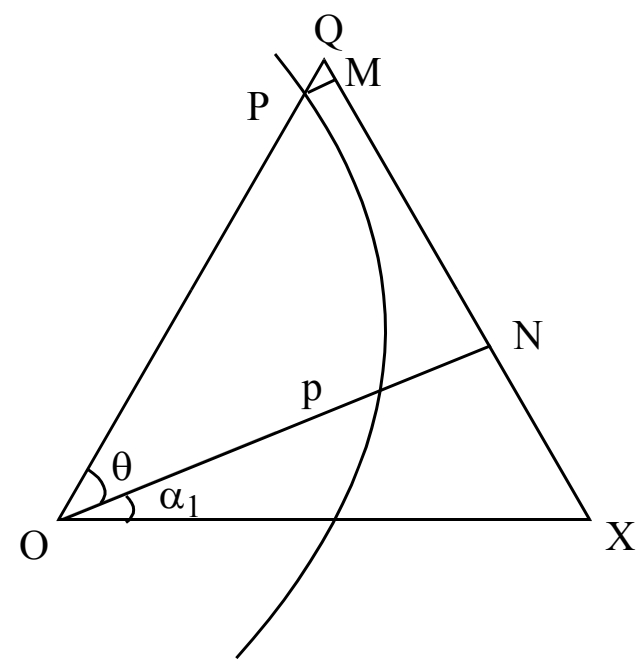

fig4 
Then $\mathrm{P}=\mathrm{ON}$, the perpendicular from the pole $\mathrm{O}$ on the line, and $\angle \mathrm{N} 0 \mathrm{X}=\alpha_{1}$. Let $\mathrm{OP}$ produced meet this line at $\theta$. The perpendicular from $\mathrm{P}$ on the line is

$\mathrm{PM}=\mathrm{PQ} \cos \theta \mathrm{PM}=(\mathrm{OQ}-\mathrm{OP}) \mathrm{Cos} \mathrm{QON}$

$$
\begin{aligned}
& =\left\{P \sec \left(\theta-\alpha_{1}\right)-f \theta_{1}\right\} \cos \left(\theta-\alpha_{1}\right) \text { from (i) and (ii) } \\
& =\mathrm{p}=\mathrm{f}(\theta) \cos \left(\theta-\alpha_{1}\right)
\end{aligned}
$$

Now, since, (ii) is any asymptote, $\mathrm{PM} \rightarrow 0$ as $\mathrm{P} \rightarrow \theta$

i.e., as $\theta \rightarrow \alpha$ for the branch in question.

$\therefore \quad \operatorname{lt}_{\theta \rightarrow \alpha}\left\{\mathrm{P}-\mathrm{f}(\theta) \cos \left(\theta-\alpha_{1}\right)\right\}=0$, or $\operatorname{lit}_{\theta \rightarrow \alpha} \mathrm{f}(\theta) \cos \left(\theta-\alpha_{1}\right)$

and as $\mathrm{P}$ is finite, and $\mathrm{f}(\theta) \rightarrow \infty$ as $\theta \rightarrow \alpha, \underset{\theta \rightarrow \alpha}{\mathrm{lt}} \cos \left(\theta-\alpha_{1}\right)=0$

$\therefore \quad \alpha-\alpha_{1}=\frac{1}{2} \pi$ or $\alpha_{1}=\alpha_{1}-1 / 2 \pi$

Again $\mathrm{P}=\operatorname{lt}_{\theta \rightarrow \alpha} \mathrm{f}(\theta) \cos \left(\theta-\alpha_{1}\right)=\operatorname{lt}_{\theta \rightarrow \alpha} \frac{\cos \left(\theta-\alpha_{1}\right)}{F(\theta)}$

which (being of the form $\left.\frac{0}{0}\right)=\operatorname{lt}_{\theta \rightarrow \alpha} \frac{-\sin \left(\theta-\alpha_{1}\right)}{F^{\prime}(\theta)}$

$=-\frac{\sin \left(\theta-\alpha_{1}\right)}{F^{\prime}(\alpha)}=\frac{1}{F^{\prime}(\alpha)}$

Hence (ii) reduces to $\mathrm{r} \cos (\theta-\alpha+12 \pi)=-1 \mathrm{f}^{\prime \prime} \alpha$

or, $\quad r \sin (\theta-\alpha)=-\frac{1}{F^{\prime}(\alpha)}$ which is the required Asymptotes.

\section{Findings:}

(1) If any values of $m$ satisfying $\phi_{n}(m)=0$ say $m=m_{1}$ make $\phi^{\prime}{ }_{n}(m)=0$ also which requires $\mathrm{m} 1$ to be multiple $\phi$ root of $\phi_{\mathrm{n}}(\mathrm{m})=0$ as we know from the theory of the equation and if $\phi_{\mathrm{n}-1}(\mathrm{~m}) \neq 0$ for this value, then $\mathrm{c} \rightarrow \infty$ as $\mathrm{m} \rightarrow$ $\mathrm{m}_{1}$. Accordingly, there is no asymptotes corresponding to this value of $\mathrm{m}$. (Simmons, George 1972)

(2) If for $\mathrm{m}=\mathrm{m}_{1}$, we get $\phi_{\mathrm{n}}(\mathrm{m}), \phi_{\mathrm{n}}{ }_{\mathrm{n}}(\mathrm{m}), \phi_{\mathrm{n}-1}(\mathrm{~m})$ each $=0$ then from equation (2) multiplying throughout by $\mathrm{x}^{2}$ and making $\mathrm{x} \rightarrow \infty$ we derive

$\frac{1}{2} \mathrm{c} 2 \phi_{\mathrm{n}}(\mathrm{m})+\mathrm{c} \phi{ }_{\mathrm{n}-1}(\mathrm{~m})+\phi_{\mathrm{n}-2}(\mathrm{~m})=0$ 
Giving two values says $c_{1}, c_{2}$ of $\mathrm{c}$ in general provided $\phi^{\prime}\left(\mathrm{m}_{1}\right) \neq 0$ and thereby giving two parallel asymptotes of the type $\mathrm{y}=\mathrm{m}_{1} \mathrm{x}+\mathrm{c}_{1}, \mathrm{y}=\mathrm{m}_{1} \mathrm{x}+\mathrm{c}_{2}$

(3) If $\phi{ }_{n}\left(m_{1)}\right.$ (is also zero, i.e. if $m$, is a triple root of $\phi_{n}(m)=0$ and

If $\phi^{\prime}{ }_{n-1}\left(m_{1}\right), \phi_{n-2}\left(m_{1}\right)$ are also identically zero, we shall proceed in a similar manner, we get three parallel asymptotes in general corresponding to $\mathrm{m}=\mathrm{m}_{1}$ and so on.

\section{Conclusion}

This article will serve as a useful introduction to the subject, through this article the student would have better understanding of the Asymptote of a curve how it looks like.

Asymptote of a curve has bee defined as a line such that the distance of any paint on the curve from this line tends to zero as the paint tends to infinity along the curve. It is believed that all the definitions of an asymptote is the one which is most natural.

This article tries to give the idea to the concept of asymptotes and its importance in teaching learning processes. Moreover, the article gives simple method for determining the asymptotes.

\section{References}

Apostol, Tom, M. (1967), Calculus Vol. 1: One-variable calculus with an introduction of linear algebra (2 ${ }^{\text {nd }}$ Ed.) New York: John Wiley and sons, ISBN 978-0-07100005-1.

Coddington, Earl. A., (1998), an Introduction of Differential Equation, (Eleventh Edition), New Delhi: Prentice Hall of India Pvt. Ltd.

D.E. Smith, History Mathematics, Vol. 2, Dover (1958), p. 318.

Das, B.C. \& Mukherjee, B.N. (1986), Differential Calculus (29 $9^{\text {th }}$ Edition), India: U.N. Dhur $\&$ Suns Private Ltd. Calcutta 700073. 
Goyal, J.K, Gupta K.P. (1999). Advance differential calculus (Fifth revised edition), Meerut: Pragati Prakashan, New Market, Begum.

Gupta, P.P., Malik GS, Mittal (2000), Differential Equation, (Sixth edition), Meerut: Pragati Prakashan.

Nunemacher, Jeffrey (1999) "Asymptote, Cubic Curves and the Projective plane" Mathematics Magazine, 72 (3): 183-192, Gte Servx 10.1.1.502.72.

Oxford English Dictionary, Second Edition, 1989.

Pant, G.D. and Shrestha, G.S. (2064), Integral Calculus and Differential Equation, Kathmandu: ABC Publication.

Shanti Narayan (Reprinted 1988), Differential Calculus. New Delhi: Shymlal Charitable Trust, Ram Nagar, -110055.

Simmons, George F. (1972), Different Equation with application and Historical Notes, Eleventh Edition, New York: McGrow Hill Publishing Company Ltd.

Singh, M.B. and Bajracharya, B.C. (2055), Differential Calculus (The second revised edition), Kathmandu: Sukunda Pustak Bhawan.

Williamson, Benjamin (1899), "Asymptotes", An elementary treaty on the differential calculus. 